\title{
O impacto da educação alimentar sobre o consumo de açúcar em alunos de escola pública da região metropolitana de Porto Alegre
}

\author{
The impact of food education sugar consumption in students \\ of metropolitan region of Porto Alegre city
}

Caroline Marques de Lima Cunha, ${ }^{1}$ Fernanda Miraglia'

'Universidade La Salle (Unilasalle), Canoas, RS, Brasil.

Recebido em: 31/07/2017 / Aceito em: 17/09/2017 / Publicado em: 01/10/2017

carol_m_/@hotmail.com

\section{RESUMO}

Os programas de educação alimentar e nutricional no ambiente escolar são fundamentais na criação, formação e modificação de hábitos alimentares de crianças e adolescentes. Objetivos: avaliar o impacto da educação alimentar sobre o consumo de açúcar na mudança de hábitos alimentares de alunos em escola pública de um município da Região Metropolitana de Porto Alegre/ RS. Método: os participantes foram avaliados antes e após a intervenção. Foram utilizados dois instrumentos: "Questionário Alimentar do Dia Anterior (QUADA-3) para escolares de seis a 11 anos" que avaliou os hábitos alimentares atuais e o "Questionário simplificado para avaliação em crianças do consumo de alimentos ricos em açúcar" para avaliação da ingestão de açúcar e bebidas açucaradas. Após, foram realizadas três oficinas educativas e interativas com intervalo de 15 dias cada. Resultados: os resultados mostraram-se estatisticamente significativos na redução do consumo de refrigerante $(p<0,05)$, frituras $(p<0,05)$ e salgadinhos ( $p<0,01)$, e no aumento da ingestão de frutas $(p<0,01)$, carnes $(p<0,05)$, pães e biscoitos $(p<0,05)$, em refeições variadas. Também se encontrou diferença estatisticamente significativa na frequência de consumo diário de balas, pirulitos e chicletes, que passou de $17,6 \%$ para $43,1 \%(p<0,01)$. Considerações finais: concluiu-se que a intervenção foi válida, pois houve redução no consumo de açúcar entre os participantes, entretanto, estas atividades de educação alimentar devem ser realizadas de forma sistemática nas escolas, a fim de incentivar e reforçar hábitos de vida saudáveis através da escolha correta de alimentos.

Palavras-chave: Educação nutricional; Ingestão alimentar; Consumo de açúcar.

\section{ABSTRACT}

Food and nutritional education programs in the school environment are fundamental in creating, formation and modification of eating habits of children and adolescents. Objectives: to evaluate the impact of nutritional education on sugar consumption in changing eating habits of students in public school in a city in the metropolitan area of Porto Alegre, state of Rio Grande do Sul. Method: subjects were evaluated for their feeding habit related mainly to the habitual consumption of sugar, through applying pre-test, which comprises using two questionnaires; after perform different activities and, in the end of the intervention it was applied a post-test, which was based on application of the same questionnaires used in the beginning. Results: the results showed statistically significant reduction in the consumption of soda, fried and salty snacks, and an increased intake of fruit, meats, breads and cookies in several meals. It was also found statistically significant difference in frequency of a once daily consumption of bullets, lollipops and chewing gum, increased from $17.6 \%$ to $43.1 \%(p<0.01)$. Results statistically not significant were also found. Closing remarks: we concluded that the intervention was positively valid; however, we suggest the realization of new more complete research, long term and with larger sample in order to encourage and strengthen healthy life habits through the correct choice of food.

Keywords: Nutrition Education; Food Intake; Sugar Consumption. 


\section{INTRODUÇÃO}

A Educação Alimentar e Nutricional (EAN) constitui uma estratégia preconizada pelas políticas públicas em alimentação e nutrição, sendo considerada um instrumento importante para promoção de escolhas alimentares saudáveis. ${ }^{1}$ Ressalta-se que os hábitos alimentares são formados na infância e que sofrem influência de fatores fisiológicos, psicológicos, socioculturais e econômicos. ${ }^{1,2}$ Também destaca-se que os mesmos se repetem ao longo do tempo e, com isso, o indivíduo sai do convívio basicamente familiar e entra no contexto escolar nessa fase da vida, na qual conhece e experimenta outros alimentos e preparações e, além de tudo, tem a oportunidade de promover alterações nas suas práticas alimentares através da influência do grupo social e dos estímulos presentes no sistema educacional. Assim, entende-se que a escola representa um ambiente extremamente favorável para programas de EAN e essa percepção vem sendo considerada na formulação de políticas públicas em alimentação e nutrição. ${ }^{3}$

A fase da infância apresenta aspectos importantes para a formação de hábitos e práticas comportamentais em geral, e especificamente alimentares. A criança, por estar inserida no contexto familiar, começa a formar e adotar os padrões de comportamento alimentar, em termos de escolha e quantidade de alimentos, horário e ambiente das refeições e, por isso, trata-se de um processo que se inicia nesta fase e estende-se por todas as demais etapas do ciclo de vida. Portanto, é ideal que seja nessa fase, caracterizada pelo constante aprendizado e conhecimento que a criança vem adquirindo, a implementação de hábitos de vida saudáveis e escolhas alimentares corretas. ${ }^{4}$

A implantação de programas de educação nutricional nas escolas, assim como a promoção de práticas alimentares e estilo de vida saudáveis nesse ambiente considerado favorável à saúde, constituem-se em importantes estratégias para enfrentar problemas alimentares e nutricionais como obesidade e doenças crônicas não transmissíveis associadas. ${ }^{1}$ Além da promoção de alimentação saudável no ambiente escolar, que parte de uma visão integral e multidisciplinar do ser humano, considera-os em seu contexto familiar, comunitário e social, a escola procura também, desenvolver conhecimentos, habilidades e agilidades para o auto-cuidado da saúde e a prevenção das condutas de risco em todas as oportunidades educativas. Além disso, estimula uma análise crítica e reflexiva sobre os valores, as condutas, as condições sociais e os estilos de vida, buscando fortalecer tudo o que contribui para melhoria da saúde e do desenvolvimento humano. ${ }^{4}$

É importante ressaltar também que a seleção de alimentos faz parte de um sistema complexo e é determinada na criança primeiramente pelos pais e por práticas culturais de seu grupo. Por isso, tornar-se ou permanecer saudável e aprender o que é necessário para um cuidado apropriado com a saúde e para a manutenção do peso, envolve metas de longo prazo, principalmente por meio de ações educativas que envolvam também os familiares dos escolares. ${ }^{1}$ Desse modo, é possível afirmar que a centralidade das práticas educativas está na transmissão de mensagens consistentes, coerentes e claras, utilizando ao máximo os recursos tecnológi- cos de comunicação, garantindo o direito ao acesso à informação. E, para colaborar com isso, os meios de comunicação atuais são modernos o suficiente para tornar o contato direto mais fácil e prático. ${ }^{5}$

Portanto, ações educacionais de caráter nutricional são benéficas em todos os sentidos, tanto para alunos quanto para escola em geral, porque a partir do momento em que o conhecimento fornecido é absorvido, escolhas e hábitos alimentares são modificados e substituídos por algo melhor nutricionalmente e, consequentemente, mais saudável. Com isso, observa-se a magnitude da educação nutricional em relação às práticas alimentares saudáveis, pois pessoas bem informadas têm mais possibilidade de participar ativamente na promoção do seu bem-estar. Nesse contexto, o estudo foi desenvolvido com o objetivo de avaliar o impacto da educação alimentar sobre o consumo de açúcar na mudança de hábitos alimentares de alunos em escola pública, de um município da Região Metropolitana de Porto Alegre.

\section{MÉTODO}

Trata-se de um ensaio semi-experimental de intervenção, realizado em escola pública de um município da Região Metropolitana de Porto Alegre/RS, durante os meses de agosto e setembro de 2014, onde foram avaliados estudantes em programa de intervenção com base em educação alimentar. Foram incluídos no estudo indivíduos de ambos os sexos, com idade entre oito e 10 anos, pertencentes, respectivamente, ao terceiro e quinto anos da escola, que fizeram a leitura assim como a assinatura do termo de assentimento livre e esclarecido (TALE), e que os pais e/ou responsáveis fizeram a leitura e assinatura do termo de consentimento livre e esclarecido (TCLE).

Setenta e cinco indivíduos foram convidados a participar do estudo, porém cinco não entraram devido ao não preenchimento do TCLE pelo responsável, e 19 não conseguiram finalizar a pesquisa devido a ausência nos dias de aplicação das atividades. Este estudo foi aprovado pelo Comitê de Ética do Unilasalle Canoas/RS (CAEE 33441114.8.0000.5307), seguindo as normas estabelecidas pela Resolução 466/2012 do Conselho Nacional de Saúde para pesquisa em seres humanos.

Os participantes (num total de $51-23$ do sexo masculino e 28 do sexo feminino) foram avaliados quanto a seu hábito alimentar relacionado principalmente ao consumo habitual de açúcar, através da aplicação de pré e pós-teste, que compreendiam a utilização de dois instrumentos: o "Questionário Alimentar do Dia Anterior (QUADA-3) para escolares de seis a 11 anos", relacionado aos hábitos alimentares atuais do participante, no qual o mesmo deveria assinalar os alimentos consumidos em cada uma das refeições realizadas no dia anterior, sendo elas: café da manhã, lanche da manhã, almoço, lanche da tarde, jantar e lanche da noite; e o "Questionário simplificado para avaliação em crianças do consumo de alimentos ricos em açúcar" que tinha como enfoque principal a ingestão de alimentos e bebidas açucaradas, pois relacionava a frequência diária ou semanal que os participantes consumiam alguns alimentos ricos em açúcar. Após o pré-teste, foram realizados três encontros quinzenais, dentro da sala de aula, com 
duração de um período de aula (45/50 minutos).

Abaixo, segue a descrição das atividades:

Atividade 1 - Construção da Pirâmide Alimentar: Objetivo: introduzir conceitos de alimentação saudável através da pirâmide alimentar. Metodologia: Após explicação da pirâmide alimentar, cada aluno recebeu três imagens diferentes de figuras de alimentos e foi solicitado que os mesmos, um de cada vez, se dirigissem até a pirâmide e colassem no local onde eles acreditassem que a respectiva imagem se encaixava. Materiais: papel Paraná, caneta, encartes de supermercado, fita dupla-face e tesoura;

Atividade 2 - Oficina com rótulos de alimentos baseada na quantidade de açúcar: Objetivo: identificar a quantidade de açúcar existente nos alimentos. Metodologia: foram distribuídas duas folhas para os alunos, sendo que uma delas continha a imagem de diversos alimentos, e a outra folha ilustrava imagens de colheres (sopa) de açúcar. Os alunos deveriam colocar ao lado de cada imagem dos alimentos, a quantidade de açúcar que eles acreditavam haver nos mesmos. Após o término, a atividade foi corrigida através da visualização dos rótulos desses alimentos com suas respectivas quantidades de açúcar. Materiais: folhas de ofício com desenhos, caneta, lápis, rótulos de alimentos com as respectivas quantidades de açúcar porcionadas em saquinhos plásticos;

Atividade 3 - Elaboração de cardápio saudável: Objetivo: conhecer alimentos que devem estar no cardápio para que o mesmo se torne completo. Metodologia: a turma, dividida em cinco grupos, elaborou um cardápio de almoço para uma semana, com colagem de figuras, e cada grupo ficou responsável pelo cardápio de um dia (segunda, terça, quarta, quinta e sexta-feira). Materiais: cinco cartolinas coloridas (uma de cada cor para a diferenciação dos dias da semana), cola, tesoura e canetinha.

Após a conclusão das atividades, foi aplicado o pós-teste que baseou-se na aplicação dos mesmos questionários iniciais, para verificar o impacto que as atividades de educação alimentar representaram, procurando analisar se houve resultado significativo da intervenção e melhora e/ou modificação dos hábitos alimentares dos participantes. As variáveis foram descritas por frequências absolutas e relativas. Para comparar o consumo alimentar, antes e após a intervenção, os testes qui-quadrado de McNemar ou Wilcoxon foram aplicados. O nível de significância adotado foi de $5 \%$ $(\mathrm{p} \leq 0,05)$ e o software utilizado para as análises estatísticas foi o SPSS (Stastistical Package for the Social Science) na versão 21.0 para Windows.

\section{RESULTADOS}

Foram incluídas no estudo 51 crianças de ambos os sexos (28 meninas e 23 meninos) entre oito e 10 anos. Na Tabela 1, apresentamos os resultados do "Questionário Alimentar do Dia Anterior (QUADA-3) para escolares de seis a 11 anos" antes e após a intervenção, demonstrando as análises encontradas e salientando as diferenças significativas e não significativas de cada segmento.
Tabela 1 - Questionário Alimentar do Dia Anterior (QUADA-3) para escolares de seis a 11 anos pré e pós-teste.

\begin{tabular}{|c|c|c|c|}
\hline Variáveis & Pré n (\%) & Pós n (\%) & $p$ \\
\hline \multicolumn{4}{|l|}{ Desjejum } \\
\hline Pães e biscoitos & $38(84,4)$ & $35(77,8)$ & 0,581 \\
\hline Achocolatado & $19(42,2)$ & $11(24,4)$ & 0,057 \\
\hline Leite e derivados & $27(60,0)$ & $25(55,6)$ & 0,804 \\
\hline Refrigerante & $10(22,2)$ & $6(13,3)$ & 0,388 \\
\hline Frutas & $8(17,8)$ & $20(44,4)$ & 0,008 \\
\hline Suco de frutas & $7(15,6)$ & $9(20,0)$ & 0,774 \\
\hline \multicolumn{4}{|l|}{ Lanche da manhã } \\
\hline Pães e biscoitos & $14(34,1)$ & $16(39,0)$ & 0,791 \\
\hline Achocolatado & $9(22,0)$ & $6(14,6)$ & 0,508 \\
\hline Leite e derivados & $11(26,8)$ & $12(29,3)$ & 1,000 \\
\hline Refrigerante & $16(39,0)$ & $9(22,0)$ & 0,118 \\
\hline Doces & $4(9,8)$ & $4(9,8)$ & 1,000 \\
\hline Salgadinhos & $10(24,4)$ & $5(12,2)$ & 0,267 \\
\hline Frituras & $6(14,6)$ & $3(7,3)$ & 0,508 \\
\hline Frutas & $17(41,5)$ & $20(48,8)$ & 0,648 \\
\hline Suco de frutas & $10(24,4)$ & $9(22,0)$ & 1,000 \\
\hline Massa & $10(24,4)$ & $5(12,2)$ & 0,180 \\
\hline \multicolumn{4}{|l|}{ Almoço } \\
\hline Arroz & $28(54,9)$ & $36(70,6)$ & 0,077 \\
\hline Refrigerante & $21(41,2)$ & $16(31,4)$ & 0,332 \\
\hline Frituras & $9(17,6)$ & $2(3,9)$ & 0,039 \\
\hline Frutas & $6(11,8)$ & $4(7,8)$ & 0,754 \\
\hline Feijão & $28(54,9)$ & $33(64,7)$ & 0,332 \\
\hline Massas & $29(56,9)$ & $21(41,2)$ & 0,134 \\
\hline Carnes & $28(54,9)$ & $37(72,5)$ & 0,022 \\
\hline Suco de frutas & $10(19,6)$ & $14(27,5)$ & 0,454 \\
\hline Verduras e legumes & $13(25,5)$ & $14(27,5)$ & 1,000 \\
\hline Sopa & $5(9,8)$ & $3(5,9)$ & 0,727 \\
\hline \multicolumn{4}{|l|}{ Lanche da tarde } \\
\hline Pães e biscoitos & $18(43,9)$ & $28(68,3)$ & 0,031 \\
\hline Achocolatado & $10(24,4)$ & $9(22,0)$ & 1,000 \\
\hline Leite e derivados & $14(34,1)$ & $19(46,3)$ & 0,302 \\
\hline Refrigerante & $15(36,6)$ & $10(24,4)$ & 0,302 \\
\hline Doces & $7(17,1)$ & $5(12,2)$ & 0,727 \\
\hline Salgadinhos & $15(36,6)$ & $6(14,6)$ & 0,012 \\
\hline Frutas & $9(22,0)$ & $9(22,0)$ & 1,000 \\
\hline Suco de frutas & $9(22,0)$ & $9(22,0)$ & 1,000 \\
\hline \multicolumn{4}{|l|}{ Jantar } \\
\hline Arroz & $21(42,0)$ & $30(60,0)$ & 0,078 \\
\hline Refrigerante & $24(48,0)$ & $13(26,0)$ & 0,019 \\
\hline Frituras & $15(30,0)$ & $13(26,0)$ & 0,804 \\
\hline Frutas & $7(14,0)$ & $4(8,0)$ & 0,508 \\
\hline Feijão & $17(34,0)$ & $23(46,0)$ & 0,210 \\
\hline Massas & $24(48,0)$ & $19(38,0)$ & 0,383 \\
\hline Carnes & $25(50,0)$ & $26(52,0)$ & 1,000 \\
\hline Suco de frutas & $9(18,0)$ & $15(30,0)$ & 0,180 \\
\hline Verduras e legumes & $8(16,0)$ & $9(18,0)$ & 1,000 \\
\hline Sopa & $8(16,0)$ & $4(8,0)$ & 0,344 \\
\hline \multicolumn{4}{|l|}{ Lanche da noite } \\
\hline Pães e biscoitos & $6(17,1)$ & $10(28,6)$ & 0,344 \\
\hline Achocolatado & $2(5,7)$ & $3(8,6)$ & 1,000 \\
\hline Leite e derivados & $15(42,9)$ & $12(34,3)$ & 0,508 \\
\hline Refrigerante & $12(34,3)$ & $12(34,3)$ & 1,000 \\
\hline Doces & $11(31,4)$ & $8(22,9)$ & 0,607 \\
\hline Salgadinhos & $9(25,7)$ & $7(20,0)$ & 0,727 \\
\hline Frutas & $11(31,4)$ & $7(20,0)$ & 0,344 \\
\hline Suco de frutas & $7(20,0)$ & $8(22,9)$ & 1,000 \\
\hline
\end{tabular}


De acordo com a tabela 1, na refeição referente ao desjejum, houve aumento estatisticamente significativo no consumo de frutas (de $17,8 \%$ para $44,4 \%$ ) e não se obteve diferença estatisticamente significativa em relação aos demais alimentos. Entretanto, a ingestão de suco de frutas obteve aumento, assim como achocolatado e refrigerante (bebidas ricas em açúcar), obtiveram diminuição no seu consumo.

Não houve diferenças estatisticamente significativas nas refeições referentes ao lanche da manhã e lanche da noite. No primeiro, houve apenas aumento não significativo na ingestão de frutas e diminuição do consumo de achocolatado, refrigerante, salgadinhos e frituras; e no segundo, houve redução não significativa no consumo de doces e salgadinhos.

$\mathrm{Na}$ refeição referente ao almoço, o consumo de carnes aumentou de 54,9\% para 72,5\% ( $p=0,022)$, e a ingestão de frituras diminuiu de $17,6 \%$ para 3,9\%; além disso, houve aumento não significativo no consumo de arroz, feijão, suco de frutas, verduras e legumes, assim como diminuição no consumo de refrigerante. Já, na refeição referente ao lanche da tarde, houve aumento estatisticamente significativo na ingestão de pães e biscoitos (de 43,9\% para 68,3\%) e diminuição estatisticamente significativa no consumo de salgadinhos (de $36,6 \%$ para $14,6 \%$ ). No jantar, houve diminuição estatisticamente significativa no consumo de refrigerante, que passou de $48,0 \%$ para $26,0 \%$.

$\mathrm{Na}$ Tabela 2, constam os resultados do "Questionário simplificado para avaliação em crianças do consumo de alimentos ricos em açúcar" antes e após a intervenção. Conforme mostra a tabela 2, diferenças estatisticamente significativas foram observadas apenas no aumento do consumo de balas, pirulitos e chicletes na frequência de uma vez por dia.

\section{DISCUSSÃO}

Através dessa pesquisa observamos que houve aumento no consumo de frutas no desjejum, e isso se deve, principalmente, ao entendimento dos participantes quanto às atividades relacionadas a escolhas alimentares saudáveis e a aplicação das mesmas no dia-a-dia de cada um. Nessa refeição, também foi possível observar que não houve diferença em relação aos demais alimentos, mesmo o suco de frutas apresentando aumento na sua ingestão, e o achocolatado e refrigerante (bebidas ricas em açúcar) demonstrando redução no seu consumo.

Nas refeições referentes ao lanche da manhã e lanche da noite, não houve diferenças e, de acordo com Levy-Costa et al., ${ }^{6}$ o consumo de refrigerante talvez não tenha se modificado significativamente devido à tendência de crescimento da ingestão dessa bebida no Brasil, e isso pode ser atribuído, principalmente, à considerável redução de preço que o torna mais acessível às populações de menor nível socioeconômico. É provável que o consumo familiar de refrigerante influencie diretamente o hábito da criança e do adolescente, o que dificultaria sua mudança quando a família não é contemplada no programa de intervenção, como ocorreu nesse estudo.

Quanto ao consumo de frutas, verduras e legumes, a proporção de crianças e adolescentes que relataram
Tabela 2 - Questionário simplificado para avaliação em crianças do consumo de alimentos ricos em açúcar antes e após a intervenção.

\begin{tabular}{|c|c|c|c|}
\hline Variáveis & Pré n (\%) & Pós n (\%) & $\mathbf{p}$ \\
\hline Achocolatado & & & 0,205 \\
\hline $1 \times /$ dia & $17(33,3)$ & $16(31,4)$ & \\
\hline $2 x /$ dia & $11(21,6)$ & $9(17,6)$ & \\
\hline $3 \times /$ dia & $7(13,7)$ & $1(2,0)$ & \\
\hline 1 a 2 x/semana & $1(2,0)$ & $5(9,8)$ & \\
\hline 3 a 4 x/semana & $0(0,0)$ & $1(2,0)$ & \\
\hline 5 a 6 x/semana & $1(2,0)$ & $2(3,9)$ & \\
\hline Nunca ou quase nunca & $14(27,5)$ & $17(33,3)$ & \\
\hline Balas, pirulitos, chicletes & & & 0,004 \\
\hline 1x/dia & $9(17,6)$ & $22(43,1)$ & \\
\hline $2 x / d i a$ & $5(9,8)$ & $6(11,8)$ & \\
\hline $3 x / d i a$ & $5(9,8)$ & $2(3,9)$ & \\
\hline 1 a $2 \times /$ semana & $5(9,8)$ & $7(13,7)$ & \\
\hline 3 a 4 x/semana & $1(2,0)$ & $0(0,0)$ & \\
\hline 5 a 6 x/semana & $10(19,6)$ & $2(3,9)$ & \\
\hline Nunca ou quase nunca & $16(31,4)$ & $12(23,5)$ & \\
\hline Bolachas recheadas & & & 0,064 \\
\hline $1 x /$ dia & $17(33,3)$ & $25(49,0)$ & \\
\hline $2 x / d i a$ & $3(5,9)$ & $3(5,9)$ & \\
\hline $3 x / d i a$ & $8(15,7)$ & $5(9,8)$ & \\
\hline 1 a 2 x/semana & $5(9,8)$ & $8(15,7)$ & \\
\hline 3 a 4 x/semana & $2(3,9)$ & 010,0 & \\
\hline 5 a 6 x/semana & $3(5,9)$ & $1(2,0)$ & \\
\hline Nunca ou quase nunca & $13(25,5)$ & $9(17,6)$ & \\
\hline Bolos ou tortas & & & 0,123 \\
\hline 1x/dia & $12(24,0)$ & $14(28,0)$ & \\
\hline $2 x / d i a$ & $6(12,0)$ & $11(22,0)$ & \\
\hline $3 x / d i a$ & $1(2,0)$ & $2(4,0)$ & \\
\hline 1 a 2 x/semana & $7(14,0)$ & $6(12,0)$ & \\
\hline 3 a 4 x/semana & $1(2,0)$ & $1(2,0)$ & \\
\hline 5 a 6 x/semana & $4(8,0)$ & $0(0,0)$ & \\
\hline Nunca ou quase nunca & $19(38,0)$ & $16(32,0)$ & \\
\hline Chocolate & & & 0,548 \\
\hline 1x/dia & $18(35,3)$ & $19(37,3)$ & \\
\hline $2 x /$ dia & $2(3,9)$ & $3(5,9)$ & \\
\hline $3 x /$ dia & $4(7,8)$ & $4(7,8)$ & \\
\hline 1 a $2 \times /$ semana & $9(17,6)$ & $7(13,7)$ & \\
\hline 3 a 4 x/semana & $2(3,9)$ & $1(2,0)$ & \\
\hline 5 a $6 \times /$ semana & $2(3,9)$ & $1(2,0)$ & \\
\hline Nunca ou quase nunca & $14(27,5)$ & $16(31,4)$ & \\
\hline Refrigerante & & & 0,458 \\
\hline 1x/dia & $12(24,0)$ & $10(20,0)$ & \\
\hline $2 \times / d i a$ & $4(8,0)$ & $8(16,0)$ & \\
\hline $3 x /$ dia & $12(24,0)$ & $8(16,0)$ & \\
\hline 1 a $2 x /$ semana & $2(4,0)$ & $6(12,0)$ & \\
\hline 3 a 4 x/semana & $1(2,0)$ & $2(4,0)$ & \\
\hline 5 a 6 x/semana & $12(24,0)$ & $6(12,0)$ & \\
\hline Nunca ou quase nunca & $7(14,0)$ & $10(20,0)$ & \\
\hline Sucos de pó ou caixinha & & & 0,364 \\
\hline $1 \times / d i a$ & $13(25,5)$ & $10(19,6)$ & \\
\hline $2 x /$ dia & $7(13,7)$ & $5(9,8)$ & \\
\hline $3 x /$ dia & $9(17,6)$ & $9(17,6)$ & \\
\hline 1 a 2 x/semana & $2(3,9)$ & $6(11,8)$ & \\
\hline 3 a 4 x/semana & $3(5,9)$ & $1(2,0)$ & \\
\hline 5 a 6 x/semana & $10(19,6)$ & $12(23,5)$ & \\
\hline Nunca ou quase nunca & $7(13,7)$ & $8(15,7)$ & \\
\hline
\end{tabular}

consumir esses alimentos foi alta nas duas refeições analisadas (almoço e jantar) no pré-teste, e manteve-se elevada pós-período de seguimento. Esses resultados assemelham-se aos achados de Neumark-Sztainer et 
al., ${ }^{7}$ que observaram a manutenção das frequências de consumo de frutas e hortaliças descritas na linha de base após a intervenção. Contudo, divergem do relatado por Gortmaker et al., ${ }^{8}$ que constataram o aumento no consumo desses alimentos entre adolescentes do sexo feminino do grupo de intervenção em comparação às do grupo controle, e de Sahota et al., ${ }^{9}$ que relataram o aumento da ingestão de porções de vegetais por adolescentes obesos submetidos à intervenção, mesmo com redução do número de porções de frutas consumidas. O consumo elevado no presente estudo desde a aplicação do pré-teste pode justificar as diferenças com os estudos mencionados, que apresentavam baixo consumo na linha de base.

Já, na refeição referente ao lanche da tarde, houve aumento na ingestão de pães e biscoitos e diminuição no consumo de salgadinhos, e isso está diretamente relacionado com as atividades trabalhadas em sala de aula que, além de incentivarem a redução do consumo de alimentos ricos em açúcar através de informações concretas, também sugeriam a substituição de alimentos industrializados (ricos em gordura e/ou sódio), por alimentos nutricionalmente mais saudáveis, melhorando assim as escolhas alimentares diárias.

No jantar, a diminuição do consumo de refrigerante foi um importante resultado, corroborando com estudos de James et al. ${ }^{10}$ e Sichieri et al.., ${ }^{11}$ os quais observaram a diminuição do consumo de refrigerante ou bebidas gaseificadas/açucaradas no grupo intervenção em comparação ao grupo controle após o período de intervenção de um ano e de sete meses, respectivamente. Nessa refeição, também se observou aumento não significativo no consumo de arroz e feijão e diminuição não significativa na ingestão de frituras, o que discorda dos resultados de um estudo transversal realizado com adolescentes com idade média de 14 anos, que remetem à hipótese de que o lanche está tomando o lugar do jantar tradicional. ${ }^{2}$ Esse fato é comprovado também em outro estudo com adolescentes universitários, em que $49 \%$ dos estudados preferiam lanchar no horário característico do jantar a realizar o jantar tradicional. ${ }^{12}$

$\mathrm{Na}$ aplicação desse questionário foi possível analisar que práticas alimentares consideradas pouco saudáveis sofreram modificações entre as crianças e adolescentes avaliados, o que sugere que comportamentos alimentares característicos dessas faixas etárias podem ser modificados mesmo com intervenções de curta duração. ${ }^{13}$ Vale ressaltar que, ao trabalharmos a alimentação baseada principalmente na redução do consumo de açúcar, também foi possível diminuir a ingestão de alimentos ricos em sódio e óleos/gorduras, como por exemplo, salgadinhos e frituras. Isso é importante, pois se torna visível que através de atividades lúdicas como essas, foi possível fornecer aos participantes informações coerentes capazes de influenciar na mudança dos hábitos alimentares diários da grande maioria deles. A atividade preferida das crianças e adolescentes foi a oficina com os rótulos dos alimentos e suas respectivas quantidades de açúcar, pois os mesmos puderam visualizar e tocar nas porções de açúcar pertencentes a cada alimento.

Em relação à tabela 2, houve aumento estatisticamente significativo no consumo de balas, pirulitos e chicletes na frequência de uma vez por dia $(p=0,004)$, que passou de $17,6 \%$ para $43,1 \%$, o que pode ser resultado da dificuldade em entender/compreender as questões propostas e suas respectivas respostas que correspondem de forma mais correta com a sua frequência de consumo do alimento em questão.

De acordo com Popkin, ${ }^{14}$ o consumo de refrigerantes, gorduras saturadas e alimentos processados tem aumentado consideravelmente nas últimas décadas em vários países. Além disso, a ingestão frequente de alimentos com alta concentração de energia (gorduras e/ou açúcares) tem sido considerada um fator importante para o aumento observado nas prevalências de sobrepeso e obesidade, assim como no incremento da predominância de diversas Doenças Crônicas Não Transmissíveis. ${ }^{15}$

Somados a isso, resultados não esperados e indesejáveis também foram encontrados após a intervenção, como a redução estatisticamente significativa do número de estudantes que consumiam balas, pirulitos, chicletes, bolachas recheadas, bolos e tortas na frequência "nunca ou quase nunca". Isso pode estar relacionado com o aumento do consumo de alimentos e bebidas açucaradas, pois nas últimas três décadas a participação do açúcar industrializado (sacarose) na dieta ultrapassou o limite de $10 \%$ recomendado pela Organização Mundial da Saúde. ${ }^{16}$ Nesse mesmo período houve também aumento na disponibilidade de embutidos (cerca de $300 \%$ ), biscoitos, açúcar e refrigerantes $(400 \%)$, o que indica uma forte tendência de aumento no consumo de produtos industrializados. ${ }^{15}$

\section{CONSIDERACÕES FINAIS}

Com base nos resultados obtidos nesse estudo, pode-se concluir que o programa de educação alimentar foi positivo, pois demonstrou impacto na mudança dos hábitos alimentares dos participantes, principalmente ao se considerar o curto tempo de intervenção e as alterações observadas na qualidade do consumo alimentar dos escolares. Baseado nos achados, acredita-se que ações educativas em nutrição devam fazer parte do currículo escolar, para que seja possível fornecer de forma clara, rápida, objetiva e frequente, informações nutricionais que auxiliem nas escolhas alimentares e estimulem a adesão e consumo de alimentos saudáveis.

\section{REFERÊNCIAS}

1. Fernandes PS, Bernardo CO, Campos RMMB, Vasconcelos FAG. Evaluating the effect of nutritional education on the prevalence of overweight/obesity and on foods eaten at primary schools. J Pediatr 2009;85(4):315-21. doi: 10.1590/ S0021-75572009000400008

2. Gambardella AMD, Frutuoso MFP, Franchi C. Prática alimentar de adolescentes. Revista de Nutrição 1999;12(1):55-63. doi: 10.1590/S1415-52731999000100005

3. Ramos FP, Santos ALS, Reis ABC. Food and nutrition education in school: a literature review. Cad Saude Publica 2013;29(11):2147-61. doi: 10.1590/0102-311x00170112

4. Yokota RTC, Vasconcelos TF, Pinheiro ARO, Schmitz BAS, Coitinho DC, Rodrigues MLCF. "Promotion of healthy eating habits by schools" study: comparison of two nutrition 
education strategies in the Federal District of Brazil. Revista de Nutrição 2010;23(1):37-47. doi: 10.1590/S141552732010000100005

5. Santos LAS. Food and nutrition education in the context of promoting healthy food practices. Revista de Nutrição 2005;18(5):681-92. doi: 10.1590/S141552732005000500011

6. Levy-Costa RB, Sichieri R, Pontes NS, Monteiro CA. Disponibilidade domiciliar de alimentos no Brasil: distribuição e evolução (1974-2003). Rev Saude Publica 2005;39(4):53040. doi: 10.1590/S0034-89102005000400003

7. Neumark-Sztainer D, Story M, Hannan PJ, Rex J. New Moves: a school-based obesity prevention program for adolescent girls. Prev Med 2003;37(1):41-51. doi: 10.1016/ S0091-7435(03)00057-4

8. Gortmaker SL, Peterson K, Wiecha J, Sobol AM, Dixit S, Fox MK, Laird N. Reducing obesity via a school-based interdisciplinary intervention among youth: Planet Health. Arch Pediatr Adolesc Med 1999;153(4):409-18. doi: 10.1001/archpedi.153.4.409

9. Sahota P, Rudolf MCJ, DixeyR, Barth JH .Randomised controlled trial of primary school based intervention to reduce risk factors for obesity. British Medical Journal 2001;323(7320):1027-29. doi: 10.1136/bmj.323.7320.1029

10. James J, Thomas P, Kerr D. Preventing childhood obesity by reducing consumption of carbonated drinks: cluster randomised controlled trial. British Medical Journal 2004;328(7450):1237-1241. doi: 10.1136/ bmj.38077.458438.EE

11. Sichieri R, Paula Trotte A, Souza RA, Veiga GV. School randomised trial on prevention of excessive weight gain by discouraging students from drinking sodas. Public Health Nutr 2009;12(2):197-202. doi: 10.1017/ S1368980008002644

12. Vieira VCR, Priore SE, Ribeiro SMR, Franceschini SCC, Almeida LP. Perfil socioeconômico, de saúde e nutrição de adolescentes recém-ingressos em uma universidade pública brasileira. Revista de Nutrição 2011;15(3):273-82 doi: 10.1590/S1415-52732002000300003

13. Vargas IC, Sichieri R, Sandre-Pereira G, da Veiga GV. Evaluation of an obesity prevention program in adolescents of public schools. Rev Saude Publica 2011;45(1):59-68. doi: 10.1590/S0034-89102011000100007

14. Popkin BM. What can public health nutritionists do to curb the epidemic of nutrition-related noncommunicable disease? Nutr Rev 2009;67Suppl 1:S79-82. doi: 10.1111/j.17534887.2009.00165.x.

15. Kac G, Sichieri R, Gigante, DP. Epidemiologia Nutricional. Rio de Janeiro: Atheneu. 2007. 580 p.

16. World Health Organization. The World Helath Report 2002: reducing risks, promoting healthy life. Geneva:WHO. 2002. $248 \mathrm{p}$.

Como citar: MARQUES DE LIMA CUNHA, Caroline; MIRAGLIA, Fernanda. O impacto da educação alimentar sobre o consumo de açúcar em alunos de escola pública da região metropolitana de Porto Alegre. Cinergis, Santa Cruz do Sul, v. 18 , n. 4 , out. 2017. ISSN 2177-4005. Disponível em: <https://online.unisc.br/seer/index.php/cinergis/article/view/10436>. Acesso em: 06 dez. 2017. doi:http://dx.doi.org/10.17058/cinergis.v18i4. 10436. 